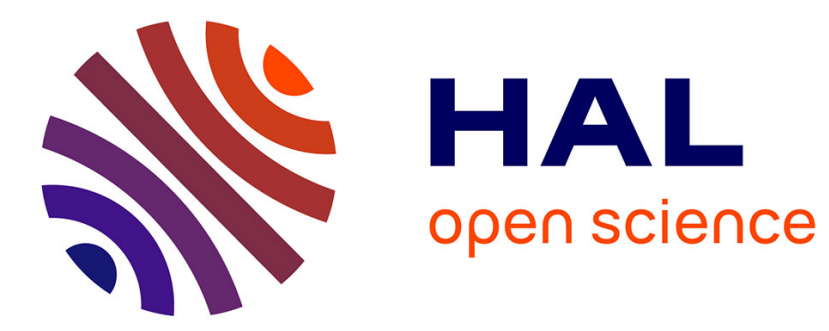

\title{
Current conduction and saturation mechanism in AlGaN/GaN ungated structures
}

J. Kuzmik, S. Bychikhin, D. Pogany, Christophe Gaquière, E. Morvan

\section{To cite this version:}

J. Kuzmik, S. Bychikhin, D. Pogany, Christophe Gaquière, E. Morvan. Current conduction and saturation mechanism in AlGaN/GaN ungated structures. Journal of Applied Physics, 2006, 99, pp.123720-1-7. 10.1063/1.2207572 . hal-00154930

\section{HAL Id: hal-00154930 \\ https://hal.science/hal-00154930}

Submitted on 22 Oct 2021

HAL is a multi-disciplinary open access archive for the deposit and dissemination of scientific research documents, whether they are published or not. The documents may come from teaching and research institutions in France or abroad, or from public or private research centers.
L'archive ouverte pluridisciplinaire HAL, est destinée au dépôt et à la diffusion de documents scientifiques de niveau recherche, publiés ou non, émanant des établissements d'enseignement et de recherche français ou étrangers, des laboratoires publics ou privés. 


\title{
Current conduction and saturation mechanism in AIGaN/GaN ungated structures
}

\author{
J. Kuzmík ${ }^{\mathrm{a})}$ \\ Institute for Solid-State Electronics, TU Vienna, Floragasse 7, A-1040 Vienna, Austria \\ and Institute of Electrical Engineering, Slovak Academy of Science, Dubravska cesta 9, \\ 84104 Bratislava, Slovakia
}

S. Bychikhin and D. Pogany

Institute for Solid-State Electronics, TU Vienna, Floragasse 7, A-1040 Vienna, Austria

C. Gaquière

IEMN/III V Lab, Common Laboratory-TIGER, Avenue Poincaré, BP 69, 59652 Villeneuve d'Ascq, France

E. Morvan

GIE Alcatel-Thales III-V Lab/TIGER, c/o Alcatel CIT, Route de Nozay, 91461 Marcoussis Cedex, France

Current conduction and saturation mechanisms in ungated $\mathrm{AlGaN} / \mathrm{GaN} / \mathrm{SiC}$ transfer length method test structures with contactto-contact distance $L$ varying from 2 to $32 \mu \mathrm{m}$ are investigated. Current-voltage $(I-V)$ characteristics are measured in the pulsed and in the direct current $(\mathrm{dc})$ regimes. The pulsed characteristics were evaluated for the pulse duration of $50 \mathrm{ns,} \mathrm{and} \mathrm{together}$ with the dc ones show that the current saturation value depends on the contact distance and the current saturation occurs at an electrical field $\ll 150 \mathrm{kV} / \mathrm{cm}$. This behavior contradicts the theoretical expectation valid for the ungated structures. An analysis of the $I-V$ characteristics shows that the dc saturation current values are up to 5.5 times lower than those under the pulsed conditions for the same $L$. If the self-heating effect was supposed to be responsible for this observation, an unrealistic temperature rise would be required. This together with the observed $\sim 1 \mathrm{~s}$ long transition time into the steady state, which is several orders of magnitude longer than what one can expect for the thermal transient, excludes the dominant role of the thermal effects in the current saturation mechanism. A model of the current conduction and saturation mechanism is suggested where the charge injection from the contacts and charging of the AlGaN surface are responsible for a AlGaN/GaN channel depletion. Thus the saturation mechanism is similar to that occurring in gated structures. The model explains well the observed dependencies and is further validated by a transient interferometric mapping method. This thermooptical method operated under the pulsed conditions allows to evaluate the free carrier concentration profiles along the structure channel and to make the comparisons with the concentration below Ohmic contacts. A strong channel depletion effect has been observed for the structure with $L=32 \mu \mathrm{m}$ in comparison to the negligible surface depletion effect found in the $L=2 \mu \mathrm{m}$ structure. This is in full agreement with the suggested model of the current saturation mechanism and with the measured $I-V$ characteristics.

\section{INTRODUCTION}

AlGaN/GaN high electron mobility transistors (HEMTs) are supposed to deliver high power and work at millimeterwave frequencies. Therefore the investigation of the electron drift and saturation velocity $\left(\nu_{\mathrm{dr}}, \nu_{\mathrm{sat}}\right.$, respectively) in the device channel is of primary importance. The value of $\nu_{\text {sat }}$ has been determined either from a HEMT small signal parameters ${ }^{1}$ or from pulsed and/or direct current (dc) currentvoltage $(I-V)$ characteristics of ungated HEMT-like structures. $^{2-8}$ In the latter case the transfer length method ${ }^{9}$ (TLM) test structure with various contact-to-contact distances have often been used for characterization.

In an ungated structure like TLM, provided that the car-

\footnotetext{
${ }^{a)}$ Author to whom correspondence should be addressed; electronic mail: jan.kuzmik@tuwien.ac.at
}

rier concentration $N$ is constant along the $\mathrm{AlGaN} / \mathrm{GaN}$ quantum well $(\mathrm{QW})$ channel and the critical electric field $E_{\mathrm{cr}}$ for the electron velocity saturation is not reached (i.e., linear regime), the current $I$ flowing between two TLM contacts can be calculated as

$$
I=e N W \mu V / L \text {, }
$$

where $e$ is the electron charge, $W$ is the channel width, $\mu$ is the low field electron mobility, $V$ is the applied voltage, and $L$ is the TLM contact distance. Monte Carlo simulations ${ }^{10}$ have predicted that electrons in $\mathrm{GaN}$ at $300 \mathrm{~K}$ reach $\nu_{\text {sat }}$ of about $3 \times 10^{7} \mathrm{~cm} / \mathrm{s}$ at $E_{\mathrm{cr}}$ of about $150 \mathrm{kV} / \mathrm{cm}$. Consequently, at high electric fields $E \geqslant E_{\text {cr }}=\nu_{\text {sat }} / \mu$ the current saturates at $I_{\text {sat }}$ :

$$
I_{\mathrm{sat}}=e N W \mu E_{\mathrm{cr}}=e N W \nu_{\mathrm{sat}},
$$

and $\nu_{\text {sat }}$ can be determined as 
TABLE I. Saturation current, saturation voltage, and critical field of the current saturation for the ungated and gated semiconductor structures with the different mechanisms of the current saturation.

\begin{tabular}{ccccc}
\hline \hline Structure & & Ungated & & \multicolumn{2}{c}{ Gated } \\
\cline { 1 - 2 } $\begin{array}{c}\text { Mechanism of } \\
\text { saturation }\end{array}$ & $\nu_{\text {sat }}$ & & $\begin{array}{c}(S) \text {-gradual } \\
\text { depletion and } v_{\text {sat }}\end{array}$ & $\begin{array}{c}(P) \text {-gradual depletion } \\
\text { and pinch-off }\end{array}$ \\
\hline$I_{\text {sat }}$ & Independent of & Dependent on & Linearly dependent on \\
& $\mu, 1 / L$ & & $\mu, 1 / L$ & $\mu / L$ \\
$V_{\text {sat }}$ & $E_{\mathrm{cr}} L$ & & $<E_{\mathrm{cr}} L$ & $V_{\mathrm{po}}-\mathrm{V}_{\mathrm{bi}}+V_{G}$ \\
$E_{\mathrm{cr}}$ & $150 \mathrm{kV} / \mathrm{cm}$ & & $<150 \mathrm{kV} / \mathrm{cm}$ & $\ll 150 \mathrm{kV} / \mathrm{cm}$ and \\
& & & linearly dependent on \\
& & & $1 / L$ \\
\hline \hline
\end{tabular}

$$
\nu_{\mathrm{sat}}=I_{\mathrm{sat}} / e N W .
$$

We emphasize that Eqs. (2) and (3) imply that the $I_{\text {sat }}$ value and the $\nu_{\text {sat }}$ determination should be independent of the TLM contact spacing $L$.

Current conduction and saturation mechanisms in gated structures [i.e., field-effect transistors (FETs)] are different from the mechanism in ungated structures. $N$ varies along the channel, and the current saturation in FET occurs either $(P)$ after the channel is gradually depleted and practically pinched off at the drain side of the channel or $(S)$ after the carrier velocity is saturated in the part of the gradually depleted channel. ${ }^{11}$ In the former case $(P)$ at the voltage onset $V^{P}$ sat of the current saturation the electric field $E$ along the channel does not reach the critical value for the electron drift velocity to saturate. $V^{P}$ sat does not depend on $L$ and can be expressed as ${ }^{11}$

$$
V_{\text {sat }}^{P}=V_{\mathrm{po}}-V_{\mathrm{bi}}+V_{G},
$$

where $V_{\text {po }}$ is the pinch-off voltage, $V_{\mathrm{bi}}$ is the built-in voltage, and $V_{G}$ is the gate bias.

On the other hand, if $E_{\mathrm{cr}}$ is reached before the channel is pinched off (case $S$ ), the current saturates at the voltage onset $V_{\text {sat }}^{S}$ and the channel is only partially depleted. That can happen if

$$
E_{\mathrm{cr}} \times L \sim V_{\mathrm{sat}}{ }^{S}<\left(V_{\mathrm{po}}-V_{\mathrm{bi}}+V_{G}\right),
$$

i.e., preferentially for short $L$. The increase of $V$ beyond $V^{S}$ sat only modulates part of the gradually depleted channel, and the channel remains opened. It is difficult to calculate $V_{\text {sat }}^{S}$ from device parameters; however $V^{S}$ sat is obviously dependent on $L$.

Because of the strong channel depletion, one can expect smaller $I_{\text {sat }}$ values for the mechanism $(P)$ than for $(S)$. The difference between case $(P)$ and $(S)$ is also manifested by the fact that for the mechanism $(P)$ one obtains a linear dependence: ${ }^{11}$

$$
I_{\text {sat }}^{P} \sim \mu / L .
$$

That is not the case of the mechanism $(S)$, where for very short $L$ Eq. (2) can be applied. The saturation mechanisms in gated and ungated structures are summarized in Table I.

Up to now the $I-V$ characterization of the ungated $\mathrm{AlGaN} / \mathrm{GaN} \mathrm{QW}$ (all with passivated surface) leads to ambiguous results, and various hypotheses were discussed in the literature..$^{2-7}$ Experiments show not only unexpected low values of $E_{\text {cr }}$ and $\nu_{\text {sat }}$, but also the questionable mechanism of the current saturation.

Vitusevich et al. reported $I-V$ experiments under the dc conditions and with $1 \mu$ s long pulses. ${ }^{2}$ On the TLM structure with $L=1-25 \mu \mathrm{m}$ a clear functional dependence of $I_{\text {sat }}$ on $L$ has been obtained. This was in contradiction with what one can expect for ungated structures ${ }^{12}$ and what Eq. (2) stipulates. Also apparent values of $E_{\mathrm{cr}}$ were $<10 \mathrm{kV} / \mathrm{cm}$. The authors assumed that the pronounced temperature rise due to Joule heating and the carrier mobility $\mu$ decrease, and not the carrier velocity saturation, account for the current saturation. However, this explanation cannot be applied for experiments with nanosecond-range pulses, where substantial elimination of the self-heating can be expected. ${ }^{13}$ Still a similar behavior ( $I_{\text {sat }}$ dependent on $L$ and/or $E_{\text {cr }} \ll 150 \mathrm{kV} / \mathrm{cm}$ ) was observed elsewhere $^{3-5}$ even for the case of very short ns-range pulses. Ardaravičius et al. $^{5}$ have examined $\mathrm{AlGaN} / \mathrm{GaN} \mathrm{QW}$ and $\mathrm{AlGaN} / \mathrm{AlN} / \mathrm{GaN}$ double-heterostructure QW samples using $1 \mathrm{~ns}$ pulses. The latter sample exhibited a perfect current saturation at $\sim 30 \mathrm{kV} / \mathrm{cm}$. The characteristics of both samples were measured up to $200 \mathrm{kV} / \mathrm{cm}$ with no differential resistance change except at $\sim 30 \mathrm{kV} / \mathrm{cm}$. Still the $\nu_{\text {sat }}$ was determined at $100 \mathrm{kV} / \mathrm{cm}$, and the value of about $(1-2) \times 10^{7} \mathrm{~cm} / \mathrm{s}$ was claimed. A Monte Carlo simulation of the hot-electron transport has shown a good agreement with the experiments only up to $30 \mathrm{kV} / \mathrm{cm}^{5}$

Different sample preparation was reported by Barker et $a l{ }^{6,7}$ Instead of the ordinary TLM structure, the authors used a special test structure providing a four-point measurement with contacts eliminating a possible carrier injection. For the case of $10 \mathrm{~ns}$ long pulses the authors reported values close to theoretical predictions: $E_{\mathrm{cr}} \sim 140 \mathrm{kV} / \mathrm{cm}$ and $\nu_{\mathrm{sat}} \sim 3.1$ $\times 10^{7} \mathrm{~cm} / \mathrm{s}$. However, an unexpected dependence of $I_{\text {sat }}$ on $L$ has also been observed, and for a different sample and $200 \mathrm{~ns}$ pulse regime the authors obtained $E_{\mathrm{cr}} \sim 30 \mathrm{kV} / \mathrm{cm}$ and $\nu_{\text {sat }} \sim 1.1 \times 10^{7} \mathrm{~cm} / \mathrm{s}$.

Elsewhere, the $\nu_{\mathrm{dr}}$ apparent saturation at about $10 \mathrm{kV} / \mathrm{cm}$ was also linked to the degradation of the AlGaN/GaN HEMT performance, ${ }^{14}$ underlining the need for a deep investigation of the current conduction and saturation mechanism.

In this work we analyze mechanisms of current saturation in AlGaN/GaN TLM structures combining dc and pulsed $I-V$ characterization with transient interferometric mapping (TIM) optical method. ${ }^{15,16}$ We assume that similarly as suggested by Hasegawa et al. ${ }^{17}$ for semi-insulating GaAs substrates (both with and without passivation of the surface), a charge injection from contacts and charge trapping on the surface may be present in AlGaN/GaN TLM structures. Consequently, the potential of the AlGaN surface is not floating as expected for the ungated structure, and we suggest a different current saturation mechanism resembling the operation of gated structures. We show that TIM results also provide an explanation of the current saturation mechanism and directly support conclusions of the $I-V$ characterization. 


\section{SAMPLE PREPARATION AND EXPERIMENTAL METHODS}

Samples were grown on 2 in. semi-insulating $4 H-\mathrm{SiC}$ substrate by metal organic chemical vapor deposition. The epilayers consist of the (from the bottom) nucleation layer, $1 \mu \mathrm{m}$ thick GaN buffer layer, $5 \mathrm{~nm}$ thick undoped AlGaN, $20 \mathrm{~nm}$ thick intentionally doped ( $\mathrm{Si}) \mathrm{AlGaN}$ and $5 \mathrm{~nm}$ thick undoped AlGaN capped with $3 \mathrm{~nm}$ undoped GaN. Thin $\mathrm{GaN}$ cap is often used in the HEMT technology to reduce the gate leakage without forming a parallel channel along the heterojunction with $\mathrm{AlGaN} .{ }^{18}$ The $\mathrm{Al}$ content was $22 \%$ and the structure was not passivated. Hall effect measurements of the epilayer performed at the room temperature indicated an electron mobility of $1300 \mathrm{~cm}^{2} / \mathrm{V} \mathrm{s}$ and a carrier concentration of $1 \times 10^{13} \mathrm{~cm}^{-2}$. A mesa isolation was defined using reactive ion etching, and the Ohmic contacts were obtained by evaporating $\mathrm{Ti} / \mathrm{Al} / \mathrm{Ni} / \mathrm{Au}$ and annealing at $900{ }^{\circ} \mathrm{C}$ in a nitrogen atmosphere. The TLM structure has a $100 \mu \mathrm{m}$ width, and the contact-to-contact distance is $L=2,4,8,16$, and $32 \mu \mathrm{m}$.

For pulsed $I-V$ characteristics, transient self-heating, and power dissipation analysis we applied a transmission line pulser (TLP) to bias the TLM contacts. A coaxial cable with a $50 \Omega$ characteristic impedance is charged by a voltage source and afterwards is discharged by closing a relay, providing thus rectangular voltage pulses. The pulse duration of $480 \mathrm{~ns}$ has been fixed by the cable length. The interval between two pulses is $\sim 0.5 \mathrm{~s}$. The current and voltage wave forms were recorded using a digital oscilloscope. To get the pulsed $I-V$ characteristics at a particular time instant averaging over several pulses in a narrow time interval was used. For the dc characterization we used a linear staircase sweep of the Keithley 2410 source meter with $\sim 40$ ms measuring time and $1 \mathrm{~s}$ delay (hold) time, the voltage step was $0.5 \mathrm{~V}$. For an additional investigation of the transient time from the pulsed into the dc state we used Agilent 8114A to generate $900 \mathrm{~ms}$ long rectangular voltage pulse.

In the TIM method the device is scanned from the backside using an infrared laser beam and synchronized with TLP pulses. The laser spot size and the TIM lateral resolution are $\sim 1.5 \mu \mathrm{m}$. The phase shift $\Delta \varphi$ of the beam reflected from the topside is caused by a temperature-induced change in the material refractive index $n$ along the beam path and is proportional to the dissipated energy in the device. ${ }^{16}$ Consequently, scanning the $\Delta \varphi$ signal provides the heat dissipation map in the device. The TLM sample is scanned with 0.5 or $1 \mu \mathrm{m}$ steps perpendicular to the structure width, and the heat spreading is recorded at selected time instances. Three measurements per step are performed, and the scanning speed is $\sim 100$ steps $/ 10 \mathrm{~min}$.

The TIM method allows also to extract the instantaneous two-dimensional power density $P_{2 \mathrm{D}}$ distribution. ${ }^{16}$ The $P_{2 \mathrm{D}}$ map serves to localize the position of the heating source. Provided that the current continuation in the TLM sample is maintained, the $P_{2 \mathrm{D}}$ distribution corresponds to the resistance profile along the device scan. In other words, the method may reveal any changes in the carrier concentration along the TLM channel and may thus visualize any channel depletion effects and/or localize the position of the high-field domain

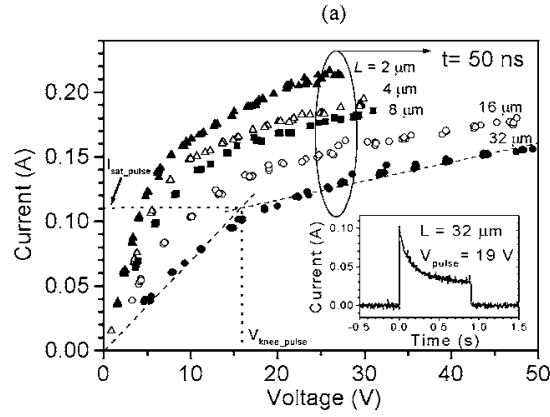

(b)

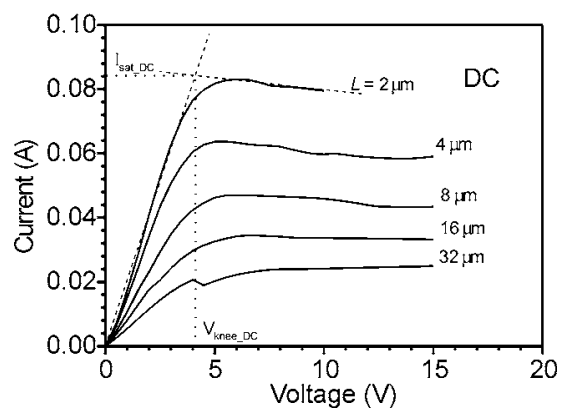

FIG. 1. I- $V$ characteristics of the TLM structure with different contact distances in (a) pulsed ( $t=50 \mathrm{~ns})$ and (b) dc conditions. Inset of (a) shows $I(t)$ transient during a $900 \mathrm{~ms} / 19 \mathrm{~V}$ pulse.

where the channel differential resistance is high. If the device structure consists of different materials (such as $\mathrm{GaN}$ on $\mathrm{SiC}$ ) with various thermo-optical coefficients, an apparent twodimensional power density $P_{2 \mathrm{DA}}$ is extracted. ${ }^{13}$ The qualitative meaning of $P_{2 \mathrm{DA}}$ is the same as $P_{2 \mathrm{D}}$; however $P_{2 \mathrm{DA}}$ reflects also the heterogeneity of the system. ${ }^{13}$ Since in our case the thermo-optical coefficients of $\mathrm{SiC}$ and $\mathrm{GaN}$ are close to each other, ${ }^{20}$ for short time instances $(t \sim 100 \mathrm{~ns})$ when the heat is located mostly in $\mathrm{GaN}$, we can assume $P_{2 \mathrm{DA}} \sim P_{2 \mathrm{D}}$.

\section{EXPERIMENTAL RESULTS AND DISCUSSIONS}

\section{A. Electrical analysis}

Figure 1 shows $I-V$ characteristics of the TLM structure under the (a) pulsed ( $t=50 \mathrm{~ns})$ and (b) dc conditions. An inset of (a) shows also a typical $I(t)$ transient characteristic during a $900 \mathrm{~ms}$ long and $19 \mathrm{~V}$ voltage pulse, indicating that the steady state is reached in the time scale of $0.1-1 \mathrm{~s}$. $I_{\text {sat }}$ is determined at $V_{\text {knee }}$ which is given by the intercepts of the linear and saturated parts of the $I-V$ characteristic (see the dc curve for $L=2 \mu \mathrm{m}$ ). The onset voltage $V_{\text {sat }}$ for the current saturation is calculated as $V_{\text {sat }}=V_{\text {knee }}-I_{\text {sat }} \times R_{C}$, where $R_{C}$ $=34 \Omega$ is the resistance of the anode plus cathode contacts determined by the TLM method. ${ }^{9} I_{\text {sat }}$ and $V_{\text {sat }}$ values are summarized in Fig. 2. The $d c$ characteristics show [see Figs. 1(b) and 2]

(i) a clear dependence of $I_{\mathrm{sat}, \mathrm{dc}}$ on $L$, with the highest $I_{\text {sat,dc }}$ values for the shortest $L$, and

(ii) a practically constant $V_{\text {sat,dc }} \sim 3 \mathrm{~V}$ for $L=8-32 \mu \mathrm{m}$.

On the other hand, the pulsed characteristics (taken at $t$ $=50 \mathrm{~ns}$ of the $480 \mathrm{~ns}$ long pulse) does not show a clear satu- 


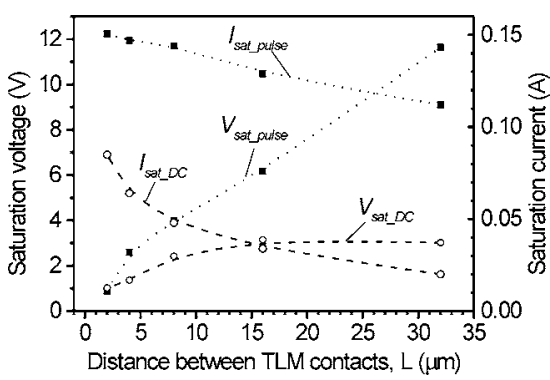

FIG. 2. Saturation current and saturation voltage in dependence on the distance between the TLM contacts for the pulsed $(t=50 \mathrm{~ns})$ and dc state, respectively.

ration in the current. This behavior is similar to what was observed for 1 and $3 \mathrm{~ns}$ pulses elsewhere ${ }^{4,5}$ for similar $\mathrm{AlGaN} / \mathrm{GaN} \mathrm{QW}$ structures. However, as mentioned in the introduction, a perfect saturation was reported for the pulsed $\mathrm{AlGaN} / \mathrm{AlN} / \mathrm{GaN}$ double-heterostructure $\mathrm{QW}$ at the same voltage bias where the $\mathrm{AlGaN} / \mathrm{GaN} \mathrm{QW}$ showed only a week saturation. ${ }^{5}$ Consequently, the current increase at $V$ $>V_{\text {knee,pulse }}$ seems to be not coupled with the current saturation mechanism and similarly as for the dc case we define the pulsed $I_{\text {sat,pulse }}$ and $V_{\text {knee,pulse }}$ directly from the intercepts of the linear and saturated parts of the $I-V$ characteristic [see Fig. 1(a)]. The reason for the week saturation is not clear. For the pulsed regime we observe

(iii) higher currents and less pronounced $I_{\text {sat,pulse }}$ dependence on $L$ in comparison to de $I-V$ and

(iv) a clear $V_{\text {sat,pulse }}$ dependence on $L$ with an almost constant $E_{\mathrm{cr}} \sim 5 \mathrm{kV} / \mathrm{cm}$.

Provided that (3) is applicable, we obtain $\nu_{\text {sat }} \sim 1$ $\times 10^{7} \mathrm{~cm} / \mathrm{s}$ at $E_{\mathrm{cr}} \sim 10 \mathrm{kV} / \mathrm{cm}$ for $L=2 \mu \mathrm{m}$ in the pulsed regime, down to $\nu_{\text {sat }} \sim 1 \times 10^{6} \mathrm{~cm} / \mathrm{s}$ at $E_{\mathrm{cr}} \sim 1 \mathrm{kV} / \mathrm{cm}$ for $L$ $=32 \mu \mathrm{m}$ under the dc condition. Those values agree with previous experiments on TLM (Refs. 2-5) but are well below values predicted theoretically. ${ }^{10}$ Similarly, the observed $I_{\text {sat }}$ vs $L$ dependencies contradict the current saturation mechanism represented by (2) and (3), and $I_{\text {sat }}$ values are well below $0.45 \mathrm{~A}$ as calculated from (2) for theoretically expected $\nu_{\text {sat }}=3 \times 10^{7} \mathrm{~cm} / \mathrm{s}^{9}$. For reference we mention also that a $0.15 \mu \mathrm{m}$ gate length HEMT processed on the identical epilayer showed similar dc characteristics as for the $L$ $=2 \mu \mathrm{m}$ TLM structure, i.e., $I_{\mathrm{sat}} \sim 0.7 \mathrm{~A} / \mathrm{mm}$ for the grounded gate, $V_{\text {knee }} \sim 3.5 \mathrm{~V}$, and $V_{\text {po }} \sim-5 \mathrm{~V}$ (not shown).

Let us discuss now a possible influence of self-heating on $I-V$ characteristics. For the case of electron velocity saturation [see (2) and (3)], only a limited change of $I_{\text {sat }}$ due to self-heating can be expected as $\nu_{\text {sat }}$ is supposed to drop by less than $10 \%$ if the temperature $T$ is increased by $\Delta T$ $=200 \mathrm{~K} .{ }^{10}$ On the other hand, if we assume that $\nu_{\text {sat }}$ is not reached, i.e., $E<E_{\text {cr }}$, Eq. (1) should be used and $I \sim \mu$. Dependencies $\mu \sim 1 / T^{\alpha}$, with $\alpha$ between 0.5 and 1.8 , have been reported. ${ }^{8,19,21}$ Consequently, more dramatic self-heating effects can be expected in the linear regime. To evaluate if the self-heating effect can be responsible for the apparent current saturation and different $I_{\text {sat }}$ values, we compare $I_{\text {sat,dc }}$ of the $32 \mu \mathrm{m}$ structure (as the limiting case) at $V=V_{\text {knee,pulse }}$
$=15.5 \mathrm{~V}$ with $I_{\text {sat,pulse }}$ at the same bias and we assume the linear regime of conduction. From the measured values (Figs. 1 and 2) we obtain $I_{\text {sat,pulse }} / I_{\text {sat,dc }}=0.11 \mathrm{~A} / 0.02 \mathrm{~A}$ $=5.5$. It was stated elsewhere ${ }^{13}$ that $\sim 17 \mathrm{~W} / \mathrm{mm}$ dissipated power density (normalized to the device width) for the $L$ $=3 \mu \mathrm{m}$ spacing will cause about $75 \mathrm{~K}$ temperature rise after 50 ns. As will be shown below (see Sec. III B), the TLM thermal resistance is decreased by a factor of $\sim 1.3$ as $L$ is increased from $2-4$ to $32 \mu \mathrm{m}$. Therefore for a structure with $L=32 \mu \mathrm{m}$ at power $P_{\text {pulse }}=17 \mathrm{~W} / \mathrm{mm}$ we can assume a temperature rise $\Delta T \sim 55 \mathrm{~K}$. On the other hand, if one wants to explain $I_{\text {sat,pulse }} / I_{\text {sat,dc }}=5.5$ solely by self-heating, $\Delta T$ values between $600 \mathrm{~K}$ and completely unrealistic $10000 \mathrm{~K}$ (depending on the $\alpha$ factor in the $\mu-T$ dependence) must be considered for the dc operation. Even the temperature increase of $600 \mathrm{~K}$ for given conditions $\left(P_{\mathrm{dc}} \sim 3 \mathrm{~W} / \mathrm{mm}, L\right.$ $=32 \mu \mathrm{m})$ is again beyond any consideration and strongly disagrees with already published observations. ${ }^{8,22}$ Similarly, as suggested by Jacquet, ${ }^{8}$ we may exclude thermal effects as a main cause of the current saturation.

\section{Model}

As shown above, none of Eqs. (1)-(3) describes the current conduction and saturation mechanism in AlGaN/GaN TLM structures properly even if the self-heating effect is considered. We assume that the main reason of that is the wrong assumption of a constant $N$ along the channel. We suggest that the AlGaN surface potential is not floating (i.e., following the channel potential), as expected for the ungated structure, ${ }^{12}$ but is biased by a charge injected from contacts. For GaAs it was reported that electrons can be injected from the cathode and subsequently trapped on the surface. ${ }^{17}$ Consequently, the potential profile along the semiconductor surface $V_{\text {surface }}(x)$ is not linearly increased from the cathode to anode, but is influenced by the presence of the surface charge. $^{17}$ If a similar mechanism is assumed for $\mathrm{AlGaN} / \mathrm{GaN}$, then $N(x)$ varies along the channel following changes in the channel-to-surface potential difference $V_{\text {channel }}(x)-V_{\text {surface }}(x)$. This effect is schematically depicted in Fig. 3, where AlGaN/GaN TLM structures with corresponding distributions of $V_{\text {channel }}(x), V_{\text {surface }}(x)$, and $N(x)$ are given for the case of the (a) floating surface potential and (b) charged surface.

In the case of the floating potential [Fig. 3(a)] the difference $V_{\text {channel }}(x)-V_{\text {surface }}(x)$ is constant and is given solely by the surface built-in potential $V_{\mathrm{bi}}$. Consequently, $N(x)$, keeps a constant value $N_{0}$, and the surface depletion effect can be neglected. However, if the electron injection/trapping from the side of the cathode occurs, an intense surface electric field at the anode edge may arise and $V_{\text {surface }}(x)$ is bent accordingly $^{17}$ [see Fig. 3(b)]. At the cathode, $V_{\text {surface }}(x)$ is fixed close to the ground potential, similarly as it was calculated using Green's function for the charged GaAs surface, ${ }^{17}$ while $V_{\text {channel }}(x)$ may increase superlinearly. ${ }^{11}$ As a result, variations in $V_{\text {channel }}(x)-V_{\text {surface }}(x)$ cause changes in $N(x)$ along the channel. We notice that the $N(x)$ distribution is also shown in the channel areas where a semiconductor surface is covered by contacts $(x>L$ and $x<0)$ and where depletion effects from the surface side can be neglected. 
(a)

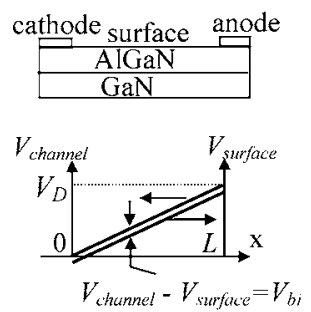

(b)
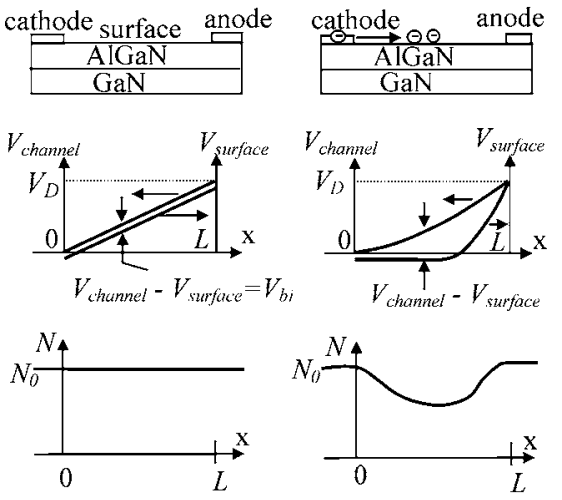

(c)

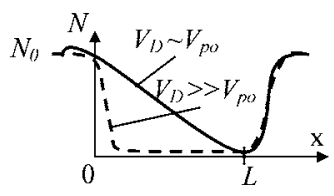

FIG. 3. Model of $\mathrm{AlGaN} / \mathrm{GaN}$ TLM structures with distributions of $V_{\text {channel }}(x), V_{\text {surface }}(x)$, and $N(x)$ for (a) floating surface potential, (b) charged surface with a moderate surface depletion, and (c) charged surface in the "pinch-off" state.

Figure 3(c) shows $N(x)$ for a case of a strong $N(x)$ modulation under the "pinch-off" condition which preferentially occur for longer $L$ [see condition (5)]. In that case the channel is initially pinched off at the anode side of the channel (full line) and may become completely pinched off as bias $V_{D}$ increases (dash line).

We stress that Fig. 3 schematically represents only one possible scenario of the charge injection and trapping. It has been reported elsewhere that charge injection into the buffer layer may take place during the HEMT breakdown. ${ }^{23}$ Thus the injection and charging also in the TLM GaN buffer layer and the channel depletion from the backside or the trapping in the $\mathrm{AlGaN}$ volume may be considered. These options will be discussed later in Sec. III B. Nevertheless, the important point is that this situation resemble the AlGaN/GaN HEMT current collapse phenomena ${ }^{24,25}$ where the presence of a virtual gate between two contacts (gate-drain) is considered. The consequences for TLM may be considerable. The TLM structure cannot be considered as an ungated structure where Eqs. (1)-(3) hold but rather resemble the model of a FET. $V_{\text {surface }}(x)$ can be understood as $V_{G}$ of the virtual gate in (4) and (5), and consequently if the charge injection/trapping is increased and $V_{\text {surface }}(x)$ is bent more effectively to $0 \mathrm{~V}$ [see Fig. 3(b)], that will decrease $V_{\text {sat }}$. To make a summary of observations (i)-(iv) we can suggest the following.

$(S 1)$. In the pulsed operation ( $t=50 \mathrm{~ns})$ the AlGaN surface becomes charged by injected charges [see (iii) and (iv) in correlation with Table I]. Structures with longer $L$ are supposed to show more signs of the pinch-off saturation mechanism (P) since for longer $L$ condition (5) is less likely fulfilled.

$(S 2)$. In the dc case the presence of the virtual gate is more pronounced (if compared with the pulsed operation.) For $L=8-32 \mu \mathrm{m}$, we can clearly assume the pinch-off saturation mechanism $(\mathrm{P})$, [see Figs. 1(b) and 2] with a clear

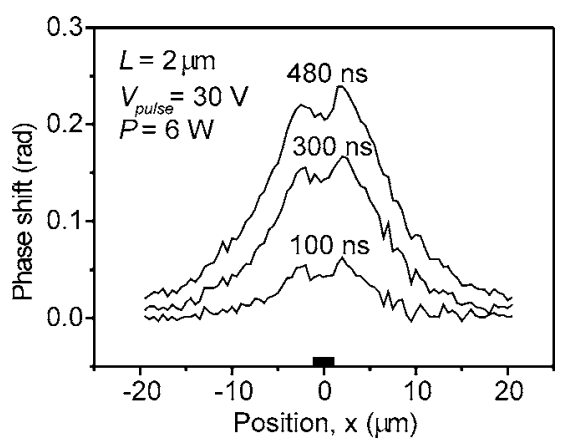

FIG. 4. TIM phase shift distribution for $L=2 \mu \mathrm{m}$ TLM stressed by $30 \mathrm{~V}$ and taken at different time instants. Contact-to-contact area is marked by a black rectangle.

dependance of $I_{\text {sat,dc }}$ on $L$ and with a practically constant $V_{\text {sat,dc. }} I_{\text {sat,dc }}$ vs $1 / L$ can be extrapolated to $0 \mathrm{~A}$ for $1 / L$ $=0 \mu \mathrm{m}$, what is not a case for $I_{\text {sat,pulse }}$ (not shown). Moreover, from (4) in this case we may obtain $V_{G} \sim-1 \mathrm{~V}$ representing the potential of the virtual gate (taking into account $V_{\text {sat,dc }} \sim 3 \mathrm{~V}, V_{\mathrm{po}} \sim 5 \mathrm{~V}$ and assuming $\left.V_{\mathrm{bi}} \sim 1 \mathrm{~V}\right)$. If one considers that for the ungated structure $V_{\text {surface }}(x) \sim(x / L) V_{D}$ [see Fig. 3(a)], then $V_{G} \sim-1 \mathrm{~V}$ represents a significant biasing of the surface.

As it was shown the surface charge trapping effect seems to be dominant in explaining the differences between the pulsed and dc characteristics, while the thermal effect is marginal. Our suggestion is clearly supported also by the time constant of the typical $I(t)$ wave form transient shown in the inset of Fig. 1. It was reported elsewhere that the time constant of the $\mathrm{AlGaN} / \mathrm{GaN}$ HEMT thermal transients is in the range of $10^{-7}-10^{-6} \mathrm{~s},{ }^{13}$ while our $I(t)$ transients indicate much longer time constants, up to $1 \mathrm{~s}$, similarly as reported for the trap-related current collapse. ${ }^{24,25}$ Consequently:

(S3). The time constant of the transition from the state of a "partially charged TLM surface" into the state of a "TLM virtual gate" may be governed by the surface trap parameters (i.e., their energy position in the AlGaN band gap and the capture cross section).

\section{B. Optical mapping analysis}

In this section we provide results of the TIM method, which were used initially to describe the heat propagation and lately to establish TLM power dissipation areas. The heat (i.e., $\Delta \varphi$ ) values were used to estimate the thermal resistance dependence on $L$ and served in Sec. III A as an input for the discussion on self-heating effects. On the other hand, $P_{2 \text { DA }}$ profiles extracted from the $\Delta \varphi$ maps will be shown to be directly linked to the mechanism of the current saturation, providing support for conclusions of Sec. III A

Figures 4 and 5 show the measured phase shift at different time instants $t$ for $L=2$ and $32 \mu \mathrm{m}$ TLM structures, respectively. The $L=2 \mu \mathrm{m}$ structure was stressed by $30 \mathrm{~V}$ pulse (power $P_{L=2}=6 \mathrm{~W}$ ), while the $32 \mu \mathrm{m}$ TLM was stressed by a higher voltage of $81 \mathrm{~V}\left(P_{L=32}=12.2 \mathrm{~W}\right)$ to keep the optical signal measurable. The $\Delta \varphi(x)$ distribution spreads and increases with time, reflecting the dynamics of the dissipated heat. Comparing $\Delta \varphi(x)$ at given $t$ of both structures, we can calculate the ratio between structure thermal resis- 


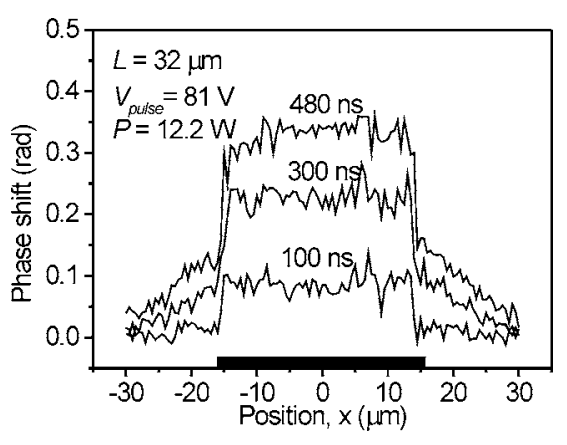

FIG. 5. TIM phase shift distribution for $L=32 \mu \mathrm{m}$ TLM stressed by $81 \mathrm{~V}$ and taken at different time instants. Contact-to-contact area is marked by the black rectangle.

tances as $\left(\Delta \varphi_{L=2} / \Delta \varphi_{L=32}\right) \times\left(P_{L=32} / P_{L=2}\right)$. In the TLM channel area (see Figs. 4 and 5) at $t=480$ ns we observe $\Delta \varphi_{L=2}$ $\sim 0.22 \mathrm{rad}$ and $\Delta \varphi_{L=32} \sim 0.34 \mathrm{rad}$. Thus the increase of $L$ from 2 to $32 \mu \mathrm{m}$ decreases the thermal resistance only by a factor of $\sim 1.3$. This is due to a less efficient cooling of the large heated area and, as will be shown below, also due to a broadening of the power dissipation area under the contacts of the $L=2 \mu \mathrm{m}$ structure.

Figures 6 and 7 show $P_{2 \mathrm{DA}}$ distributions calculated from profiles of Figs. 4 and 5 at $t=100 \mathrm{~ns}$. In the calculation procedure $^{13}$ we used $d n / d T=5.2 \times 10^{-5} \mathrm{~K}^{-1}$, other GaN material parameters are listed elsewhere. ${ }^{13}$ We use $P_{2 \mathrm{DA}}$ distributions to establish the length of the heat dissipation area $L_{H}$ defined as a full width at half maximum of $P_{2 \mathrm{DA}}(x)$ distributions (see Figs. 6 and 7). For the $L=32 \mu \mathrm{m}$ TLM structure we obtained $L_{H} \sim 30 \mu \mathrm{m} \sim L$; however, for $L=2 \mu \mathrm{m}$ we see $L_{H} \sim 12 \mu \mathrm{m} \gg L$. For a further understanding of this discrepancy we compare "electrical" values of $P_{2 \mathrm{DE}}$ calculated from the $I-V$ wave forms as

$$
P_{2 \mathrm{DE}}=(I \times V) /(L \times W),
$$

with $P_{2 \text { DA }}$ obtained from TIM. A good agreement was obtained for $L=32 \mu \mathrm{m}$ as $P_{2 \mathrm{DA}} \sim 4.5 \mathrm{~mW} / \mu \mathrm{m}^{2} \sim P_{2 \mathrm{DE}}$; however, for $L=2 \mu \mathrm{m}$ one sees a disagreement as $P_{2 \mathrm{DA}}$ $\sim 4.6 \mathrm{~mW} / \mu \mathrm{m}^{2} \ll P_{2 \mathrm{DE}} \sim 30 \mathrm{~mW} / \mu \mathrm{m}^{2}$. A good coincidence can only be obtained if in Eq. (7) the contact distance $L$ $=2 \mu \mathrm{m}$ is replaced with the "experimental" dissipation length $L_{H} \sim 12 \mu \mathrm{m}$. This confirms our experimental observations that the heat dissipation region of the short TLM is not defined by the contact distance but overlaps the contact area.

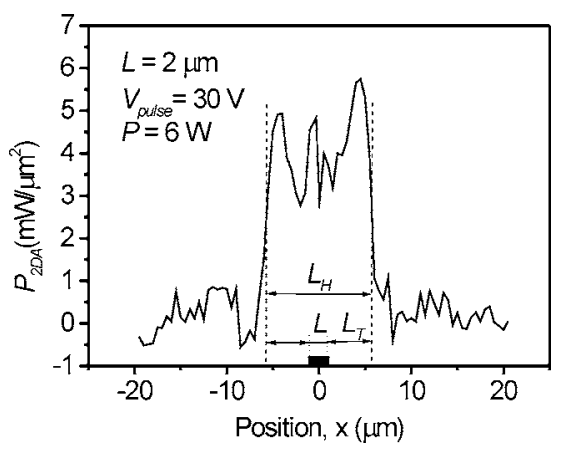

FIG. 6. Apparent two-dimensional power density determined by TIM for $L=2 \mu \mathrm{m}$ TLM stressed by $30 \mathrm{~V}$.

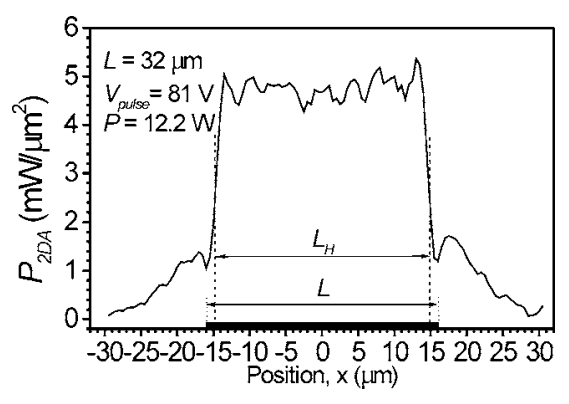

FIG. 7. Apparent two-dimensional power density determined by TIM for $L=32 \mu \mathrm{m}$ TLM stressed by $81 \mathrm{~V}$.

The difference $L_{H}-L$ can easily be interpreted as two times the transfer length $L_{T}$ of the Ohmic contacts (see Fig. 6):

$$
L_{H}-L=2 L_{T}
$$

as $L_{T}$ is the "effective" part of the contact where the current is crowded. ${ }^{9}$ To confirm this hypothesis we determined $L_{T}$ directly from the TLM using the electrical method. ${ }^{9}$ The TLM experimental procedure was performed in the linear regime (applying $0.5 \mathrm{~V}$ ), thus minimizing $V_{\text {channel }}(x)$ $-V_{\text {surface }}(x)$ and possible channel depletion effects. The method (see Fig. 8) leads to $2 L_{T} \sim 11.5 \mu \mathrm{m}$, confirming our interpretations.

Our final discussion concerns the fact that even though $L_{T}$ as a technological parameter is not supposed to be dependent on $L$, contacts are not "visible" in the $L=32 \mu \mathrm{m}$ structure (see Fig. 7). Some power dissipation below contacts of the $L=32 \mu \mathrm{m}$ structure can be recognized, still in this case in the sense of (8) $L_{T}=0 \mu \mathrm{m}$. This can be explained by a substantial depletion of the channel in the $L=32 \mu \mathrm{m}$ structure below the charged free surface, as illustrated by a dashed line in Fig. 3(c) and by the restoration of $N_{0}$ under the contacts. Power dissipates preferentially at the position of the maximal resistance, and, consequently, for $L=32 \mu \mathrm{m}$ maximal $P_{2 \mathrm{DA}}$ is confined between the contacts. On the other hand, Fig. 6 indicates that the resistance of the channel below the free surface of the $L=2 \mu \mathrm{m}$ structure is comparable to the resistance of the channel below the contacts, and this resembles the situation illustrated in Fig. 3(b) where only a small decrease of $N$ below contacts [if compared with Fig. 3(c)] is expected. Consequently, a comparable power is dissipated both in the channel and contact regions, so according to the definition of $L_{H}$ we observe $L_{H}>L$ and $L_{T}$ can be determined from (8). We note that our observations do not imply that

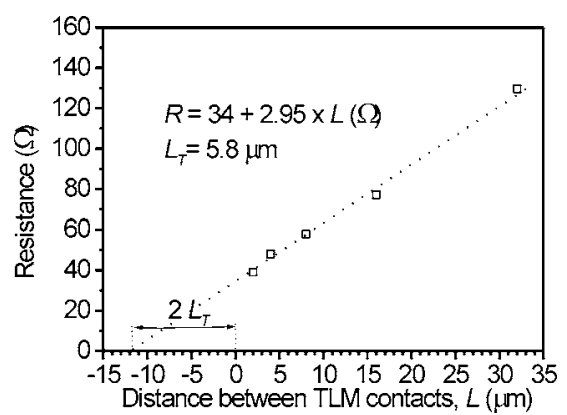

FIG. 8. Dependence of the TLM structure resistance on the contact distance and extraction of the transfer length. 
carrier injection and trapping are enhanced with increasing $L$, but in agreement with (5) we show that if the sample surface is charged, the pinch-off saturation mechanism (and strong channel depletion) appears preferentially for longer TLM structures. Our explanation is in full agreement with suggestion (S1), and thus results of our optical characterization strongly support conclusions derived from the electrical characterization. Finally, we discuss if the charge injection/ trapping is dominantly present at the sample surface, as assumed in the text above, or if the charging of the GaN buffer below the channel and/or in the AlGaN may also be considered to be decisive. If the charge injection/trapping was present dominantly at the buffer/channel interface or in the $\mathrm{AlGaN}$, this should take place along the whole conductive path of electrons, i.e., also below the contacts along the transfer length $L_{T}$. Consequently, in this case, even if the channel is being depleted we should expect $L_{H} \sim L+L_{T}$. However, this assumption contradicts present experimental observations (see Fig. 7). Our experimental results show a sharp restriction of the heat dissipation area of the $L$ $=32 \mu \mathrm{m}$ structure by the device contacts (see Fig. 7). Thus the charge injection/trapping and the channel depletion are restricted by contact edges, and we may suggest that depletion takes place dominantly from the side of the surface. This is valid at least for the case of the strong channel depletion [current saturation mechanism $(P)$ ] before a possible channel breakdown.

\section{CONCLUSIONS}

We have investigated the current conduction and saturation mechanism in the AlGaN/GaN ungated TLM structures using electrical and optical mapping methods. We have suggested that the early saturation in TLM $I-V$ characteristics and the determined low apparent electron saturation velocity are the consequence of the injection of charges from contacts, surface charging, and channel depletion from the side of the surface. A model explaining the potential and carrier distribution in the channel and on the AlGaN surface has been proposed. This model is strongly supported by the TIM measurements, which allow us to identify the channel depletion effects. It has been shown that the studied TLM structures cannot be described as conventional ungated devices but resemble devices with a virtual gate. It has been explained that the transition from the pulsed $I-V$ characteristic into the dc one is governed mainly by the surface charging, and thermal effects are marginal.

\section{ACKNOWLEDGMENTS}

This work was performed in the context of the network TARGET, "Top Amplifier Research Groups in a European Team" (www.target-org.net), and of the project ULTRAGAN, "InAlN/(In)GaN Heterostructure Technology for Ultra-High Power Microwave Transistors." It was supported by the Information Society Technologies Program of the EU under Contract Nos. IST-1-507893-NOE and 006903. Support and discussions with J.-P. Teyssier are also acknowledged.

${ }^{1}$ C. H. Oxley and M. J. Uren, IEEE Trans. Electron Devices 52, 165 (2005).

${ }^{2}$ S. A. Vitusevich et al., Appl. Phys. Lett. 82, 748 (2003).

${ }^{3}$ B. A. Danilichenko et al., Appl. Phys. Lett. 85, 5421 (2004).

${ }^{4}$ L. Ardaravičius, A. Matulionis, J. Liberis, O. Kiprijanovic, M. Ramonas,

L. F. Eastman, J. R. Shealy, and A. Vertiatchikh, Appl. Phys. Lett. 83, 4038 (2003)

${ }^{5}$ L. Ardaravičius et al., Phys. Status Solidi A 202, 808 (2005).

${ }^{6}$ J. M. Barker, D. K. Ferry, S. M. Goodnick, D. D. Koleske, A. Allerman, and R. J. Shul, J. Vac. Sci. Technol. B 22, 2045 (2004).

${ }^{7}$ J. M. Barker, D. K. Ferry, S. M. Goodnick, D. D. Koleske, A. Allerman, and R. J. Shul, Phys. Status Solidi C 2, 2564 (2005).

${ }^{8}$ J.-C. Jacquet, European Microwave Week 2004 Conference Proceedings, Amsterdam 2004 (Horizon House, London, 2004), pp. 235-238.

${ }^{9}$ D. K. Schroder, Semiconductor Materials and Device Characterization (Wiley, New York, 1998), p. 156.

${ }^{10}$ U. Bhapkar and M. S. Shur, J. Appl. Phys. 82, 1649 (1997).

${ }^{11}$ M. Shur, GaAs Devices and Circuits (Plenum, New York, 1987), p. 301.

${ }^{12}$ M. Shur, GaAs Devices and Circuits (Plenum, New York, 1987), p. 358.

${ }^{13}$ J. Kuzmík, S. Bychikhin, M. Neuburger, A. Dadgar, A. Krost, E. Kohn, and D. Pogany, IEEE Trans. Electron Devices 52, 1698 (2005).

${ }^{14}$ T. Palacios, S. Rajan, A. Chakraborty, S. Heikman, S. Keller, S. P. DenBaars, and U. K. Mishra, IEEE Trans. Electron Devices 52, 2117 (2005).

${ }^{15}$ D. Pogany, S. Bychikhin, Ch. Fürböck, M. Litzenberger, E. Gornik, G. Groos, K. Esmark, and M. Stecher, IEEE Trans. Electron Devices 49, 2070 (2002)

${ }^{16}$ D. Pogany, S. Bychikhin, M. Litzenberger, G. Groos, and M. Stecher, Appl. Phys. Lett. 81, 2881 (2002).

${ }^{17}$ H. Hasegawa, T. Kitagawa, T. Sawada, and H. Ohno, Electron. Lett. 20, 561 (1984)

${ }^{18}$ H. Zhang and E. T. Yu, J. Appl. Phys. 99, 014501 (2006).

${ }^{19}$ J. R. Shealy, V. Kaper, V. Tilak, T. Prunty, J. A. Smart, B. Green, and L. F. Eastman, J. Phys.: Condens. Matter 14, 3499 (2002).

${ }^{20}$ P. J. L. Hervé and L. K. J. Vandamme, J. Appl. Phys. 77, 5476 (1995).

${ }^{21}$ I. Daumiller, C. Kirchner, M. Kamp, K. J. Ebeling, and E. Kohn, IEEE Electron Device Lett. 20, 448 (1999).

${ }^{22}$ J. Kuzmik, P. Javorka, A. Alam, M. Marso, M. Heuken, and P. Kordoš, IEEE Trans. Electron Devices 49, 1496 (2002).

${ }^{23}$ J. Kuzmík, D. Pogany, E. Gornik, P. Javorka, and P. Kordoš, Appl. Phys. Lett. 83, 4655 (2003).

${ }^{24}$ I. Daumiller et al., IEEE Electron Device Lett. 22, 62 (2001).

${ }^{25}$ G. Meneghesso, G. Verzellesi, R. Pierobon, F. Rampazzo, A. Chini, U. K. Mishra, C. Canali, and E. Zanoni, IEEE Trans. Electron Devices 51, 1554 (2004). 